\title{
MODELAGEM DA GESTÃO DE SEGURANÇA EM LABORATÓRIO DE PESQUISA E ENSINO
}

\author{
Sarah Francisca de Souza Borges \\ Instituto Tecnológico da Aeronáutica \\ Praça Marechal Eduardo Gomes, 50, Vila das Acácias, CEP 12228-900, São José dos \\ Campos, SP, Brasil \\ sarah@ita.br \\ Marco Antônio Fontoura de Albuquerque \\ Instituto Tecnológico da Aeronáutica \\ Praça Marechal Eduardo Gomes, 50, Vila das Acácias, CEP 12228-900, São José dos \\ Campos, SP, Brasil \\ mafa@ita.br \\ Moacyr Machado Cardoso Junior \\ Instituto Tecnológico da Aeronáutica \\ Praça Marechal Eduardo Gomes, 50, Vila das Acácias, CEP 12228-900, São José dos \\ Campos, SP, Brasil \\ moacyr@ita.br \\ Mischel Carmen Neyra Belderrain \\ Instituto Tecnológico da Aeronáutica \\ Praça Marechal Eduardo Gomes, 50, Vila das Acácias, CEP 12228-900, São José dos \\ Campos, SP, Brasil \\ carmen@ita.br \\ Luís Eduardo Loures da Costa \\ Instituto Tecnológico da Aeronáutica \\ Praça Marechal Eduardo Gomes, 50, Vila das Acácias, CEP 12228-900, São José dos \\ Campos, SP, Brasil \\ loures@ita.br
}

\section{RESUMO}

O Brasil investiu em projetos que possibilitam o desenvolvimento e o lançamento de satélites com diferentes finalidades, minimizando a dependência dos países fornecedores e ampliando o conhecimento nacional. Devido ao cenário e perigos existentes, o objetivo deste estudo é avaliar as barreiras de controle e mitigação de perigos em laboratórios acadêmicos. Foram analisados os métodos Soft System Methodology (SSM), para obter uma visão holística da situação problemática, definição das principais transformações e, identificação de perigos; Hazard and Effect Management Process (HEMP) e Bowtie para definir as barreiras de prevenção e mitigação de perigos. A contribuição do trabalho reside na integração dos métodos mencionados. Não foram encontrados trabalhos semelhantes em uma pesquisa bibliográfica na base de dados Scopus e Web of Science. Alguns dos 
resultados obtidos foram adoção de algumas barreiras, como: plano de resposta a emergências, controle de acesso e treinamento de segurança de técnicos do laboratório.

Palavra-chave: Estruturação de Problemas. Gerenciamento e Análise de Perigos. Segurança em Laboratórios.

\section{ANALYSIS OF TOOLS FOR MODELING SECURITY MANAGEMENT IN RESEARCH AND TEACHING LABORATORIES}

Brazil has invested in projects that enable the development and launch of satellites with different purposes, minimizing dependence on supplier countries and increasing national knowledge. Due to the existing scenario and dangers, the objective of this study is to evaluate the hazard control and mitigation barriers in academic laboratories. The Soft System Methodology (SSM) methods were analyzed to obtain a holistic view of the problematic situation, definition of the main transformations and identification of hazards; Hazard and Effect Management Process (HEMP) and Bowtie to define the barriers of prevention and mitigation of hazards. The contribution of the work lies in the integration of the mentioned methods. No similar work was found in a bibliographic search in the Scopus and Web of Science database. Some of the results obtained were the adoption of some barriers, such as: emergency response plan, access control and safety training of laboratory technicians.

Keywords: Problem Structuring; Hazard Analysis and Management; Laboratory Safety.

\section{Como Citar:}

BORGES, Sarah Francisca de Souza; ALBUQUERQUE, Marco Antônio Fontoura de. JUNIOR, Moacyr Machado Cardoso; BELDERRAIN, Mischel Carmen Neyra; COSTA, Luís Eduardo Loures da. Modelagem da Gestão de Segurança em Laboratório de Pesquisa e Ensino. In: SIMPÓSIO DE PESQUISA OPERACIONAL E LOGÍSTICA DA MARINHA, 19., 2019, Rio de Janeiro, RJ. Anais [...]. Rio de Janeiro: Centro de Análises de Sistemas Navais, 2019.

\section{INTRODUÇÃO}

O Brasil tem investido em projetos que viabilizam o desenvolvimento e lançamento de satélites com diversos propósitos, minimizando a dependência dos países fornecedores e ampliando resultados nacionais. Os estudos e testes desenvolvidos nos laboratórios acadêmicos do ITA contribuirão para que o país efetue os lançamentos de satélites em seu território e a propriedade intelectual nacionalizada dos produtos gerados permitirá vencer barreiras tecnológicas.

Seguindo esses propósitos, os valores estratégicos do Instituto Tecnológico de Aeronáutica (ITA), como instituição de educação e ensino superior, estão fundamentados na busca permanente pela excelência, com foco no aluno e ensino de qualidade. Para tal, admitir laboratórios que estejam absolutamente alinhados ao cenário atual, atendendo aos desafios mercadológicos e acadêmicos, alinha as necessárias demandas da prática com as inovações [1].

Para tanto, a implementação da prevenção de acidentes deve envolver os diversos segmentos acadêmicos responsáveis pelo processo da atividade de ensino. E, frente ao 
cenário existente e riscos presentes, o objetivo deste estudo é realizar uma análise metodológica para avaliar as barreiras de segurança do Laboratório de Sistemas de Injeção para Propulsores Líquidos do ITA. Para tanto, dentro de cada âmbito, foram selecionados os métodos: Soft System Methodology (SSM) para análise do contexto inserido, atores envolvidos e visão do problema de maneira holística; Hazard and Effect Management Process (HEMP) para identificação de perigos, eventos potenciais e suas consequências; e Bowtie para identificação de barreiras ou ações de segurança. Finalmente, diversas contribuições apoiam a sinalização do cenário ideal e são propostas barreiras preventivas e de mitigação de perigos para o Laboratório. Destaca-se a motivação de garantir a segurança necessária, reduzindo os perigos de danos a pessoas, meio ambiente, patrimônio e reputação, além do atendimento aos requisitos legais vigentes.

\section{REVISÃO DA LITERATURA}

Trabalhos em laboratórios requerem o conhecimento dos riscos existentes, as barreiras de segurança existentes e ações que contribuam para melhoria. Regras básicas de segurança em laboratórios e recomendações gerais para o desenvolvimento de um trabalho experimental seguro estão principalmente relacionadas com qualquer Instituição de Ensino. Isso significa que o tempo dedicado ao planejamento das atividades desenvolvidas no laboratório contribui na prevenção de riscos químicos, biológicos e de acidentes com a manipulação de equipamentos.

Ao considerar a Soft System Methodology ou Metodologia de sistemas flexíveis ou SSM uma abordagem prática [2], em função de sua perspectiva orientada para a ação, para a compreensão de questões complexas, e com a finalidade de aprendizado e ação, é possível contribuir para melhoria de processos já existentes no Laboratório de Sistemas de Injeção para Propulsores Líquidos (CEPROS). Com base neste mapeamento sistêmico, foi possível identificar possíveis áreas críticas para o bom funcionamento do laboratório acadêmico em questão nos padrões e requisitos legais em vigor.

Além disso, em paralelo aos passos da SSM, para verificação das principais transformações necessárias, foram utilizadas as metodologias de análise de perigos HEMP e Bowtie que apoiam na resolução da situação problemática. O método Bowtie facilita por ser uma ferramenta de exibição gráfica da relação entre os vários componentes que geram o perigo e pode ser resultado da prévia aplicação do método HEMP.

A origem do diagrama Bowtie é desconhecida, pois muitos registos foram perdidos ao longo do tempo. Acredita-se que originalmente se denominava "diagrama borboleta" e que tenha evoluído para diagramas de causa/consequência na década de 1970. A primeira referência a esta metodologia apareceu na Universidade de Queensland, Austrália, na data de 1979. Além disso, a metodologia amadureceu na década de 90 após o desastre de Piper Alpha, na plataforma de petróleo no mar do Norte. Por fim, em 1999, por meio de um projeto do Grupo Shell, uma nova abordagem foi concebida centrada no acontecimento crítico, denominada HEMP, agrupada ao diagrama Bowtie, essas ferramentas permitem identificar os perigos e analisar as barreiras necessárias [3, 4].

Assim, risco é o efeito da incerteza nos objetivos, sua gestão pode ser aplicada a toda uma organização, em suas várias áreas e níveis, a qualquer momento, abrangendo funções, atividades e projetos específicos. Além disso, possibilita a organização: aumentar a probabilidade de atingir objetivos; encorajar uma gestão pró-ativa; melhorar a identificação de oportunidades e ameaças; entre outros [5].

De acordo com a norma regulamentadora [6], Programa de Prevenção de Riscos Ambientais (PPRA), os riscos devem ser avaliados de forma abrangente, dentro de cinco 
grupos principais: riscos de acidentes, ergonômicos, físicos, químicos e biológicos. Preconiza como riscos ambientais, os agentes físicos, químicos e biológicos existentes nos ambientes de trabalho, que em função de sua natureza, concentração ou intensidade no tempo de exposição, podem causar danos à saúde do trabalhador.

As Diretrizes Curriculares Nacionais (DCN) do MEC para os Cursos de Graduação em Engenharia, instituída pela Resolução CNE/CES 11, de 11 de março de 2002, define em seu Art. $5^{\circ}$ no parágrafo $2^{\circ}$ "Nos conteúdos de Física, Química e Informática, que é obrigatório a existência de procedimentos de laboratório. Nos demais conteúdos básicos, deverão ser previstas atividades práticas e de laboratórios, com enfoques e intensidades compatíveis com a modalidade pleiteada” [7].

Os laboratórios acadêmicos possuem um papel fundamental, tanto nas atividades de ensino, pesquisa, como na prestação de serviços. Desta forma, fica evidente que reunir os mais adequados equipamentos e ter absoluto controle operacional sobre o mesmo se faz necessário por meio principalmente do estabelecimento de controle das barreiras de segurança, bem como dos processos de qualidade na entrega final de produtos e serviços [8].

Desta forma, o tempo dedicado ao desenvolvimento de uma gestão de riscos, com todas as suas etapas e níveis de abrangência, possibilitarão que a exposição seja consideravelmente minimizada, obedecendo as normas e procedimentos de segurança para trabalhos em laboratórios. Quando se pensa em riscos em laboratórios, existe a tendência do pensamento em perigos específicos da atividade fim, sem contemplar uma visão mais abrangente, considerando os impactos ambientais, pessoas, ativos e reputacionais.

\section{INTEGRAÇÃO DE MÉTODOS} aplicação.

Neste capítulo serão descritos os métodos selecionados, dentro de cada âmbito de

\subsection{SOFT SYSTEM METHODOLOGY (SSM)}

A metodologia SSM ou Metodologia dos Sistemas Flexíveis foi desenvolvida no período de 1969 a 1972, por Peter Checkland na Universidade de Lancaster com o objetivo de apoiar na resolução de problemas administrativos reais, aplicando-se ideias sistêmicas a situações reais e usando a experiência adquirida para modificar tais ideias e sua metodologia de utilização [9].

Trata-se de um método de estruturação de problemas (dentro da Pesquisa Operacional Soft), com enfoque sistêmico para a criação de modelos conceituais, que ajudem na compreensão da situação problemática e apoiem na identificação de ações de melhoria. De modo geral, a SSM permite a ação em um cenário complexo, por meio da comparação entre um modelo conceitual e os elaborados por observações do ambiente em que o problema está inserido, ou seja, o que realmente ocorre na situação estudada. Esses sistemas são construídos não apenas através da observação dos fatos e da lógica da situação, mas também a partir dos pontos de vista de todos os atores envolvidos no processo. É uma metodologia composta por sete estágios e sua aplicação ocorre principalmente com a definição das essências do sistema relevante, identificando os elementos da palavra mnemônica CATWOE (C - clientes; $\mathrm{A}$ - atores internos e externos; $\mathrm{T}$ - transformações necessárias; W - visão global; O - donos e E - restrições do ambiente) e o estabelecimento dos modelos conceituais $[9,11]$. 


\subsection{HAZARD AND EFFECT MANAGEMENT PROCESS (HEMP)}

Com base na análise com SSM, é possível compreender o problema em questão, bem como identificar os perigos existentes. Assim, é possível a aplicação adequada de métodos de análise de risco. A metodologia HEMP é utilizada para análise e identificação prévia de ameaças, para propor medidas de segurança (Barreiras de Controle-BC e Barreiras de Mitigação-BM), que impeçam a ocorrência dos eventos iniciais. Em caso de um evento, a operação visa, rapidamente, estabelecer a normalidade das operações e controle de suas consequências [13].

Inicialmente, o perigo é identificado e a consequência da liberação do perigo é estabelecida. Em seguida, pode-se agrupar a outro método de avaliação de risco (probabilidade e gravidade) de uma lesão a pessoal e danos a ativos, ambiente ou reputação.

A estratégia de mitigação especificada pelo HEMP é determinada pela identificação de mais perigos, com respectivas consequências e barreiras. Claramente, a eliminação definitiva de um perigo é o método ideal de mitigação de risco. Contudo, essa via de mitigação de risco está frequentemente fora do escopo de detalhes de revisões de segurança do processo. Além disso, as avaliações de segurança deveriam receber foco logo no design do projeto, em oposição ao raciocínio da existência do perigo para posterior ação cautelar $[12,13]$.

\subsection{BOWTIE}

Após a análise pelo método HEMP e os resultados obtidos, é possível selecionar cada perigo para estudo e representação por meio da técnica Bowtie (Gravata Borboleta). Essa técnica é uma representação gráfica para análise de como um perigo pode ser liberado, quais as causas de ocorrência do evento principal e suas consequências. Pode ser considerada uma ferramenta de gerenciamento de perigos, permitindo descrever a complexa relação entre perigos, ameaças, consequências, barreiras de controle, bem como contribuir para a identificação das atividades críticas [12, 14].

\section{RESULTADOS E DISCUSSÃO}

Neste capítulo serão descritos os resultados encontrados e pontos de discussão.

\subsection{LABORATÓRIO CEPROS}

Em 2012, pesquisadores do ITA, Instituto de Estudos Avançados (IEAv), Instituto de Aeronáutica e Espaço (IAE) e Instituto Nacional de Pesquisas Espaciais (INPE) criaram uma rede de cooperação na área de propulsão líquida. A equipe gestora é composta de profissionais com experiência e conhecimento em motores foguetes, combustão, física, química, e parceiras de várias empresas.

Além disso, a formação desta rede de cooperação nos Institutos Nacionais de Ciência e Tecnologia surge como ocasião oportuna à necessidade de aproximação e conexão de diferentes equipes (pesquisadores do ITA, IEAv, IAE e INPE), que trabalham no desenvolvimento de sistemas propulsivos e de maximização de seu potencial. Tais equipes cooperam seja na formação e capacitação de recursos humanos especializados, seja no compartilhamento de suas experiências laboratoriais.

\subsection{APLICAÇÃO DA SSM}

Com base nas 7 primeiras etapas da metodologia SSM proposta por Checkland (1981), a seguir, serão apresentados os principais conteúdos gerados. 


\subsubsection{Explorar a situação problemática}

O estudo de caso é descrito a seguir com base em entrevistas com os professores, principais responsáveis pelo Laboratório em questão, considerada uma situação carente de fatos claros, de modo que, o planejamento sistêmico de recursos e orientações pode resultar na melhor estruturação de atividades, alinhamento entre os principais atores e motivação para investimentos.

O Laboratório CEPROS conta com o principal apoio de professores, alunos e pesquisadores do ITA e busca prever o comportamento de componentes de uma câmara de combustão de motor foguete a propelente líquido. Neste projeto há necessidade de estudos na parte de identificação de perigos do Laboratório, pois já existe o Laboratório CEU (Câmara de Celula Única) que realiza estes mesmos experimentos, mas em escala menor, atualmente em uso por orientandos dos professores dos Institutos citados. O Laboratório CEPROS ainda está vigente e conta com a infraestrutura pronta, mas falta a compra de equipamentos, contratação de pessoal de apoio (técnicos) para atuar no Laboratório, roteiros de ensino para disciplinas e ainda há espaço para muitas sugestões de gestão de atividades e perigos. Além disso, atualmente, os professores atuam no planejamento dos procedimentos do Laboratório CEPROS, com atenção à segurança e relatórios para devida prestação de contas à Financiadora, e tendo como base a análise de risco pelo método HAZOP (BARBOSA; AMILCAR, 2018).

\subsubsection{Expressar a situação problemática}

Para expressar a situação problemática pela SSM é proposto a utilização de uma Figura Rica (apresentada na Figura 1, a seguir). Figuras Ricas são um ponto de partida ideal para lidar com situações desordenadas, buscando-se capturar tudo o que se pretende conhecer sobre a situação desordenada sem impor nenhuma estrutura ou análise, lançando uma imagem "descoberta". Retrata todos os fatores envolvidos, ideias, pessoas, estruturas; enfim, toda a situação que está se analisando. Além disso, podem ser representados elementos subjetivos, como: características, sentimentos, conflitos e preconceitos (literalmente "pré-julgamentos") [17]. 


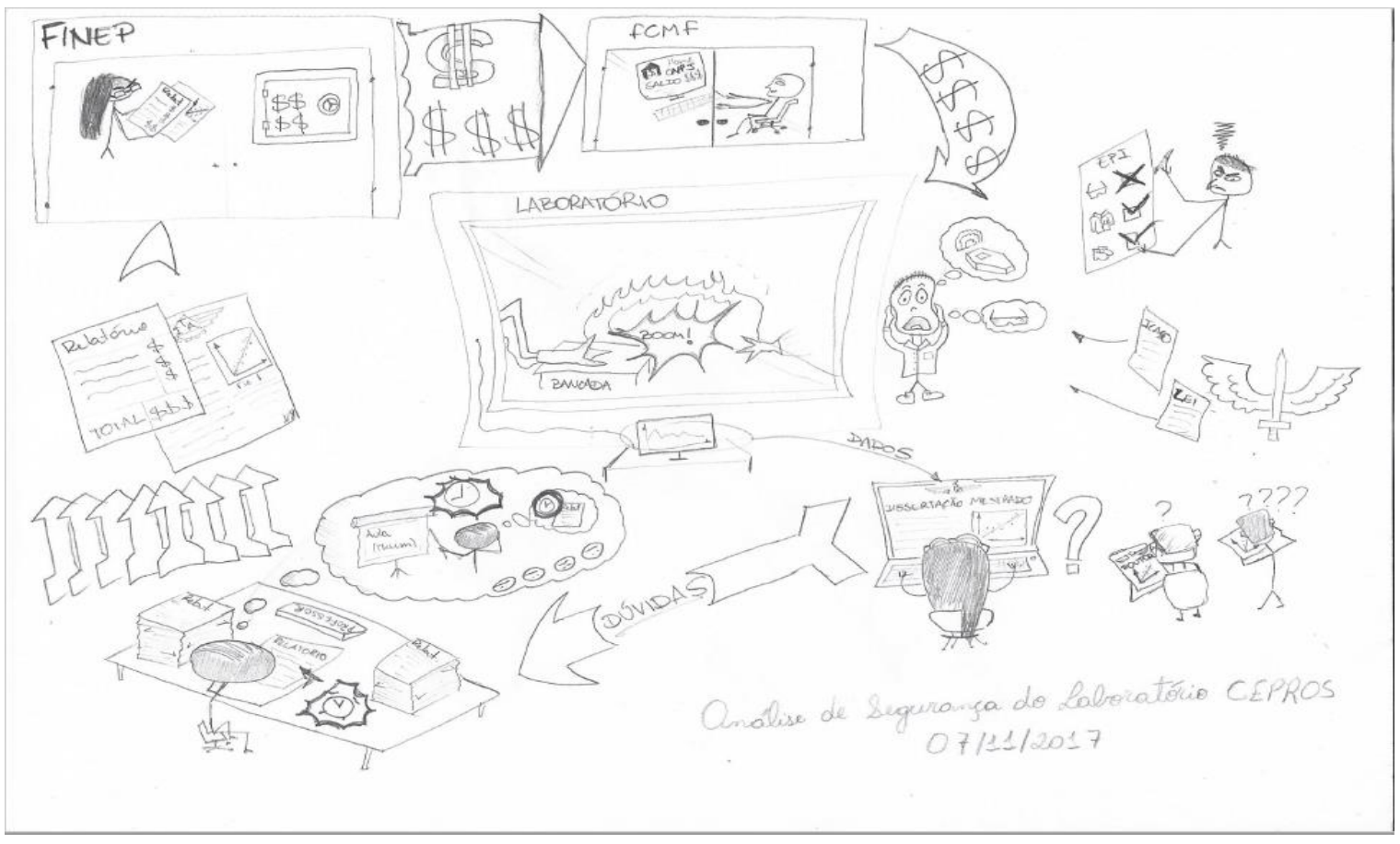

Figura 1 - Figura Rica

\subsubsection{Construir definições sucintas de sistemas relevantes}

Neste estágio são identificados os sistemas relevantes (SR) da SSM [15, 17] utilizando a abordagem CATWOE, cada Transformação (T) deve possuir um CATWOE (conforme Quadros 1 e 2). Além disso, é válido ressaltar que se buscou resumir em 2 (duas) transformações, sendo que cada uma implica em uma definição raiz, ou seja, significa dizer o que deve ser feito, como deve ser feito e por que deve ser feito. SSM "Construir definições sucintas (ou raiz) de sistemas relevantes”.

\begin{tabular}{|l|l|}
\hline CLIENTES (CLIENT) & Estudantes e pesquisadores atuantes no Projeto do Laboratório. \\
\hline ATORES (ACTORS) & $\begin{array}{l}\text { Professores, técnicos, alunos (graduação e pós-graduação), } \\
\text { Segurança do Trabalho do ITA. } \\
\text { Outros atores: Financiadora, Ministério da Ciência, Tecnologia, } \\
\text { Inovações e Comunicações (MCTIC), Empresas Fornecedoras } \\
\text { de tanque pressurizado. }\end{array}$ \\
\hline $\begin{array}{l}\text { TRANSFORMAÇÕES } \\
\text { (TRANSFORMATION) }\end{array}$ & $\begin{array}{l}\text { Alta exposição aos riscos no Laboratório. } \rightarrow \text { Baixa exposição } \\
\text { aos riscos no Laboratório. }\end{array}$ \\
\hline VISÃO DO MUNDO & $\begin{array}{l}\text { Os laboratórios universitários são ambientes de trabalho cujos } \\
\text { objetivos estão voltados para o ensino, pesquisa e extensão. } \\
\text { Assim, a abordagem de segurança sob os aspectos da lei, } \\
\text { normas e procedimentos contribui para conscientização dos } \\
\text { docentes e discentes, em relação a prática preventiva. }\end{array}$ \\
\hline
\end{tabular}




\begin{tabular}{|c|c|}
\hline $\begin{array}{l}\text { PROPRIETÁRIOS } \\
\text { (OWNER) }\end{array}$ & Comando da Aeronáutica e Reitoria do ITA. \\
\hline $\begin{array}{l}\text { RESTRIÇÕES } \\
\text { AMBIENTAIS } \\
\text { (ENVIROMENTAL } \\
\text { CONSTRAINTS) }\end{array}$ & $\begin{array}{l}\text { - Total de recurso financeiro disponibilizado pela FINEP. } \\
\text {-Alinhamento do cronograma de atividades, composto pela } \\
\text { compra de equipamentos, contratação de técnicos, estruturação e } \\
\text { planejamento das atividades, ensaios e tratamento de resultados. } \\
\text { - Plano de segurança para operação, de modo a mitigar riscos, } \\
\text { treinamento de pessoal e atendimento a normas técnicas. }\end{array}$ \\
\hline \multicolumn{2}{|c|}{$\begin{array}{l}\text { Definição raiz: Um sistema que atenda estudantes e pesquisadores; operado por professores, } \\
\text { técnicos, alunos (graduação e pós-graduação) e segurança do trabalho do ITA; que passa da alta } \\
\text { a baixa exposição aos riscos do laboratório; em função da abordagem de segurança sob os } \\
\text { aspectos da lei, normas e procedimentos que contribuem para conscientização dos docentes e } \\
\text { discentes; pertencentes ao Comando da Aeronáutica e Reitoria do ITA; e opera obedecendo ao } \\
\text { total de recurso financeiro, alinhamento do cronograma de atividades e plano de segurança para } \\
\text { operação. }\end{array}$} \\
\hline
\end{tabular}

Quadro 1 - CATWOE do Sistema Relevante 1: Exposição de pessoal aos riscos no Laboratório CEPROS.

\begin{tabular}{|l|l|}
\hline CLIENTES (CLIENT) & Estudantes e pesquisadores atuantes no Projeto do Laboratório. \\
\hline ATORES (ACTORS) & $\begin{array}{l}\text { Professores, técnicos, alunos (graduação e pós-graduação), } \\
\text { Segurança do Trabalho do ITA. } \\
\text { Outros atores: Financiadora, Ministério da Ciência, Tecnologia, } \\
\text { Inovações e Comunicações (MCTIC), Empresas Fornecedoras de } \\
\text { tanque pressurizado. }\end{array}$ \\
\hline $\begin{array}{l}\text { TRANSFORMAÇÕES } \\
\text { (TRANSFORMATION) }\end{array}$ & $\begin{array}{l}\text { Baixa dedicação de professores e técnicos no planejamento de } \\
\text { atividades do Laboratório. } \rightarrow \text { Alta dedicação de professores e } \\
\text { técnicos no planejamento de atividades do Laboratório. }\end{array}$ \\
\hline $\begin{array}{l}\text { VISÃO DO MUNDO } \\
\text { (WELTANSCHAUUNG } \\
\text { ) }\end{array}$ & $\begin{array}{l}\text { Os laboratórios universitários são ambientes de trabalho cujos } \\
\text { objetivos estão voltados para o ensino, pesquisa e extensão. Assim, } \\
\text { a abordagem de segurança sob os aspectos da lei, normas e } \\
\text { procedimentos contribui para conscientização dos docentes e } \\
\text { discentes, em relação a prática preventiva. }\end{array}$ \\
\hline $\begin{array}{l}\text { PROPRIETÁRIOS } \\
\text { (OWNER) }\end{array}$ & \begin{tabular}{l} 
Comando da Aeronáutica e Reitoria do ITA. \\
\hline RESTRIÇÕES
\end{tabular} \\
\hline
\end{tabular}


AMBIENTAIS

(ENVIROMENTAL

CONSTRAINTS)
-Alinhamento do cronograma de atividades, composto pela compra de equipamentos, contratação de técnicos, estruturação e planejamento das atividades, ensaios e tratamento de resultados.

- Plano de segurança para operação, de modo a mitigar riscos, treinamento de pessoal e atendimento a normas técnicas.

Definição raiz: Um sistema que atenda estudantes e pesquisadores; operado por professores, técnicos, alunos (graduação e pós-graduação) e segurança do trabalho do ITA; que passa da baixa a alta dedicação de professores e técnicos no planejamento de atividades do Laboratório; em função da abordagem de segurança sob os aspectos da lei, normas e procedimentos que contribuem para conscientização dos docentes e discentes; pertencentes ao Comando da Aeronáutica e Reitoria do ITA; e opera obedecendo ao total de recurso financeiro, alinhamento do cronograma de atividades e plano de segurança para operação.

Quadro 2 - CATWOE do Sistema Relevante 2: Planejamento antecipado e eficaz das atividades do Laboratório CEPROS.

\subsubsection{Elaborar modelos conceituais}

Nesta etapa, foram elaborados modelos conceituais para estruturação das atividades viáveis e necessárias para as transformações:

1. Gerenciamento de riscos eficiente e eficaz, com base em ferramentas e técnicas conceituadas, para mitigação da exposição de pessoal à possibilidade de explosões no Laboratório.

2. Planejamento antecipado das atividades para controle de tarefas e cumprimento de objetivos de projetos no Laboratório, seguindo as normas legais vigentes e requisitos acordados entre a Financiadora e os professores, ao final, desenvolvimento de estudos que agreguem valor à Instituição.

Como os modelos conceituais devem ter critérios de controle complementares e específicos, baseado no estudo realizado e CATWOE, foi montado o Sistema de Atividade Humana (Human Activity System - HAS). Sendo que, o Quadro 3 apresenta os 5 (cinco) critérios de controle propostos para cada Transformação deste sistema.

\begin{tabular}{|c|c|c|c|}
\hline $\begin{array}{c}\text { Critérios de } \\
\text { Controle }\end{array}$ & Transformação & Atividade & Foco \\
\hline \multirow{2}{*}{ Eficácia } & 1 & 1 & $\begin{array}{c}\text { Reduzir o registro de acidentes no } \\
\text { Laboratório. }\end{array}$ \\
\cline { 2 - 4 } & 2 & 2 & $\begin{array}{c}\text { Atingir resultado esperado com controle e } \\
\text { qualidade. }\end{array}$ \\
\hline \multirow{2}{*}{ Eficiência } & 1 & 3 & $\begin{array}{c}\text { Planejar mitigação de riscos e periódica } \\
\text { atualização de planos de ação em caso de } \\
\end{array}$ \\
\cline { 2 - 4 } & 2 & 4 & $\begin{array}{c}\text { Planejar atividades antecipadamente, com } \\
\text { menores custos e roteiros estabelecidos. }\end{array}$ \\
\hline
\end{tabular}




\begin{tabular}{|c|c|c|c|}
\hline \multirow{2}{*}{ Efetividade } & 1 & 5 & $\begin{array}{l}\text { Utilizar o Laboratório de forma segura e } \\
\text { consciente. }\end{array}$ \\
\hline & 2 & 6 & $\begin{array}{l}\text { Atender satisfatoriamente as demandas para } \\
\text { utilização do Laboratório. }\end{array}$ \\
\hline \multirow{2}{*}{ Ética } & 1 & 7 & $\begin{array}{l}\text { Estar o Laboratório e sua operação de acordo } \\
\text { com normas e leis. }\end{array}$ \\
\hline & 2 & 8 & $\begin{array}{l}\text { Estar o Laboratório e sua operação de acordo } \\
\text { com normas da Instituição de Ensino. }\end{array}$ \\
\hline \multirow{2}{*}{ Elegância } & 1 & 9 & $\begin{array}{l}\text { Permitir no intervalo de tempo um } \\
\text { procedimento simplificado para atender a } \\
\text { demanda urgente. }\end{array}$ \\
\hline & 2 & 10 & $\begin{array}{l}\text { Permitir no intervalo de tempo um } \\
\text { procedimento completo para atender a } \\
\text { demanda quando necessário. }\end{array}$ \\
\hline
\end{tabular}

Quadro 3 - Sistema de Atividades Humanas (HAS).

Uma vez que as respectivas HASs foram projetadas e acordadas para Transformação, elas estão unidas para formar o plano sistêmico geral que deve ser seguido para resolver a situação. A Figura 2 apresenta uma ilustração de relação entre as atividades do sistema.
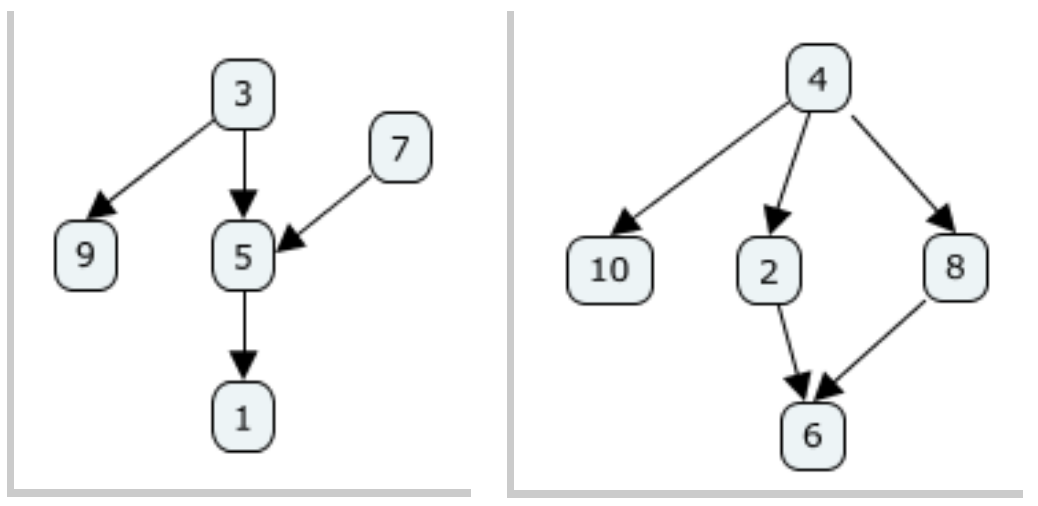

Figura 2 - Modelo Conceitual para Transformação 1 e 2 respectivamente.

Após este processo, é possível montar um supersistema com a união de vários HAS, conforme apresentado na Figura 3, e considera-se que as atividades deverão ser monitoradas por meio de critérios de controle (eficácia, eficiência, efetividade, ética e elegância). 


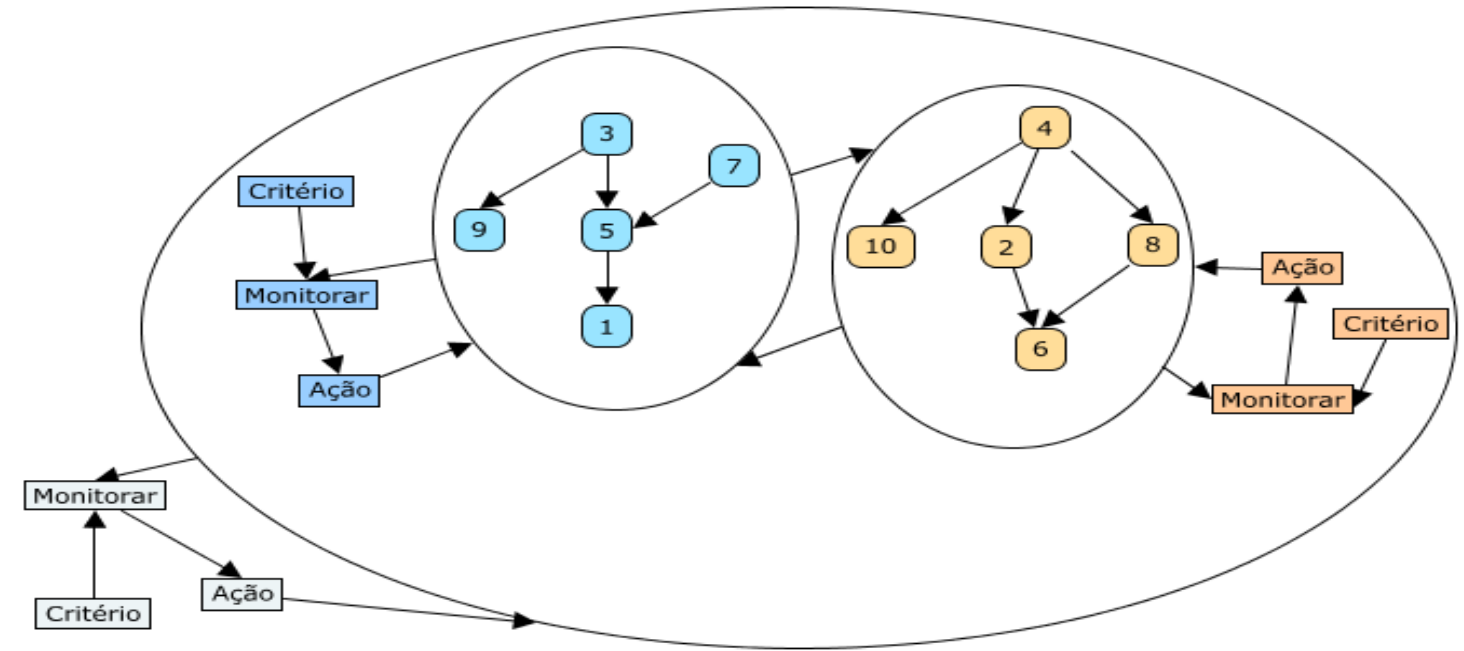

Figura 3 - Modelo Conceitual Agregado

\subsubsection{Comparação da etapa 4 com etapa 2}

Após a identificação das atividades são selecionadas aquelas que possam ser executadas sem depender de outras, sendo as atividades 3, 7 e 4 independentes. Para a Transformação 1, as atividades 9 e 5 dependem da atividade 3, e a atividade 1 depende da atividade 5. Já para Transformação 2, as atividades 10, 2 e 8 dependem da atividade 4, e a atividade 6 depende das atividades 2 e 8 conjuntamente.

Comparando-se o mundo real com os modelos de sistemas de atividade é possível verificar as diversas interações entre os Stakeholders ou conjunto de interessados, apoiando na identificação das mudanças possíveis, como discriminado a seguir.

\subsubsection{Listar mudanças possíveis e desejadas}

Transformação 1: Exposição de pessoal à riscos de explosão no Laboratório.

- Utilização de ferramentas para medição do nível de risco atual do Laboratório;

- Identificação e classificação de perigos no Laboratório;

- Mitigação e controle de riscos no Laboratório.

Transformação 2: Planejamento completo e eficaz das atividades do Laboratório.

- Definição de roteiros de atividades a serem realizadas no Laboratório;

- Plano de ação para atender às demandas do Laboratório com segurança e eficácia.

- Capacitação ou contratação de profissionais capacitados para desenvolver os procedimentos e dar a atenção necessária aos equipamentos envolvidos no Laboratório (por exemplo, técnico deverá comunicar aos professores responsáveis no caso de alguma válvula não estar funcionando corretamente).

- Teste periódico de equipamento e válvulas do Laboratório, evitando que ocorram falhas nos ensaios.

\subsubsection{Sugerir ações para a transformação da situação problemática}

Para as duas transformações é listado, a seguir, as tarefas vistas como essenciais para as melhorias.

- Planejamento antecipado das atividades a serem realizadas no Laboratório (com roteiros e responsáveis bem definidos); 
- Estudo financeiro de quanto a gestão do Laboratório precisará embolsar ou solicitar a Financiadora em equipamentos de proteção individual (EPIs) e demais equipamentos, materiais e ferramentas voltadas para segurança.

- Desenvolver plano de emergência em caso de acidente, bem como disponibilizar instruções claras e visíveis no Laboratório, realizando treinamentos e atualizações periodicamente ao material pelos professores e técnicos.

- Constante atenção sobre a disponibilidade de materiais e EPIs no Laboratório. Aprimoramento de equipamentos e sistemas de software com instruções claras e compreensíveis, de modo que, os técnicos do laboratório e qualquer indivíduo consiga desligar o sistema caso necessário.

- Desenvolver um manual de treinamento para todos os stakeholders, incluindo as políticas, regras e procedimentos operacionais, garantindo o conhecimento e a capacitação de todos os usuários, a cada início de atividades acadêmicas ou projetos específicos.

\subsection{APLICAÇÃo DO HEMP}

Assim, a grande preocupação verificada em laboratórios universitários nos cursos de engenharia é devido à presença de diversos riscos. Para que as atividades nos laboratórios sejam executadas com segurança são necessárias que medidas preventivas sejam efetivas. A seguir, são apresentados alguns resultados da aplicação do HEMP (Quadro 4), abrangendo o estudo de caso do Laboratório CEPROS, com base em entrevistas com os professores responsáveis. 


\begin{tabular}{|c|c|c|c|c|}
\hline \multicolumn{5}{|c|}{$\begin{array}{c}\text { HAZARD AND EFFECT MANAGEMENT PROCESS - HEMP } \\
\text { REGISTRO DE ANÁLISE DA ATIVIDADES }\end{array}$} \\
\hline \multicolumn{5}{|c|}{ Unidade / Setor:' Laboratório de Sistemas de Injeção para Propulsores Líquidos } \\
\hline PERIGOS & AMEAÇAS & EVENTOS PRINCIPAIS & BARREIRAS & CONSEQUÊNCIA / IMPACTOS \\
\hline Gases & $\begin{array}{l}\text { Falha humana } \\
\text { Falta de cuidado na Área de Estoque } \\
\text { Falha/ausência de procedimento } \\
\text { Falha na operação de equipamentos } \\
\text { Equipamento Obsoletos }\end{array}$ & Perda de Contenção & $\begin{array}{l}\text { Mapa de Riscos = BC } \\
\text { Utilizaçãa de EPIs do Laboratório = BC } \\
\text { Guila de Procedimentos atualizados e dirulgados = BC } \\
\text { Sinalização de Segurança = BC } \\
\text { Exames Médicos Admissionais e Periódicos = BC } \\
\text { Confinamento dos Cilindros = BC } \\
\text { Estabelecer Programa de Treinamento = BC } \\
\text { Plano de Atendimento emergencial = BM }\end{array}$ & $\begin{array}{l}\text { Fatalidade / Lesões } \\
\text { Perdas Financeiras } \\
\text { Descontinuidade Operacional }\end{array}$ \\
\hline $\begin{array}{l}\text { Equipamentose } \\
\quad \text { Válvulas }\end{array}$ & $\begin{array}{l}\text { Falhalausência de procedimentos } \\
\text { Falha humana (fadiga, pressão, ....) } \\
\text { Falha no material: } \\
\text { - corrosãa } \\
\text { - desgaste } \\
\text { - impacto } \\
\text { - acoplamento de peças } \\
\text { - partes energizadas } \\
\text { - desregulagem } \\
\text { Ausêncialdeficiência de sinalização }\end{array}$ & \begin{tabular}{|l} 
Queimadura \\
Explosão \\
Choque elétrico \\
Queda \\
Tropeços \\
Impacto
\end{tabular} & $\begin{array}{l}\text { Guia de Procedimentos atualizados e diullgados = BC } \\
\text { Estabelecer Programa de Treinamento = BC } \\
\text { Projeto adequado = BC } \\
\text { Especificação correta de material = BC } \\
\text { Programa de manutenção = BC } \\
\text { Proteção das partes móveis = BC } \\
\text { Utilização de EPIs do Laboratório = BC } \\
\text { Proteção e sinalizaçãa das partes energizzadas = BC } \\
\text { Estabelecimento de check list diário e semestral = BC } \\
\text { Plano de Atendimento emergencial = BM } \\
\text { Isolamento / Sinalização de Segurança = BC }\end{array}$ & $\begin{array}{l}\text { Lesões Leves } \\
\text { Lesões Graves } \\
\text { Descontinuidade Operacional } \\
\text { Fatalidade } \\
\text { Perdas Financeiras }\end{array}$ \\
\hline Ergonômico & $\begin{array}{l}\text { Falha/ausência de procedimento } \\
\text { Falha humana } \\
\text { Falta de projetos } \\
\text { Equipamentos impróprios }\end{array}$ & $\begin{array}{l}\text { Posição desconfortável } \\
\text { Mesma posição por horas } \\
\text { Executar atividades sob pressão } \\
\text { Excesso de peso } \\
\text { Licença médica: } \\
\text { - Lesão músculo-esquelético } \\
\text { - Dor localizada }\end{array}$ & $\begin{array}{l}\text { Exames Médicos Admissionais e Periodicos Atualizados = BC } \\
\text { Estabelecer Programa de Treinamento = BC } \\
\text { Estabelecimento de check list diário e semestral }=\text { BC } \\
\text { Utilização de EPIs Ergonômicos no Laboratório = BC } \\
\text { Plano de Atendimento emergencial = BM }\end{array}$ & $\begin{array}{l}\text { Lesão grave (músculo-esquelético) } \\
\text { Descontinuidade Operacional }\end{array}$ \\
\hline Sabotagem & $\begin{array}{l}\text { Falha/ausência de procedimento } \\
\text { Falha humana } \\
\text { Falha no controle de acesso }\end{array}$ & $\begin{array}{l}\text { Interuppção parcial das atividades } \\
\text { Dano à Imagem }\end{array}$ & $\begin{array}{l}\text { Câmeras de Segurannça = BC } \\
\text { Estabelecer Programa de Treinamento = BC } \\
\text { Estabelecimento de check list diário e semestral }=\text { BC } \\
\text { Plano de Atendimento emergencial = BM } \\
\text { Controle de acesso ao laboratório = BC }\end{array}$ & \begin{tabular}{|l|} 
Danos ao patrimônio \\
Danos a reputação \\
Interrupção total das atividades \\
Fatalidade / Lesões
\end{tabular} \\
\hline
\end{tabular}

Quadro 4-HEMP

\subsection{APLICAÇÃo BoWTIE}

Como o Bowtie pode ser montado para representação de um perigo por vez, visando o estudo de causas, consequências e medidas de mitigação antes e após a ocorrência, foi realizado a análise do acidente com “explosão" (Figura 4), considerado no nível de severidade mais crítica. Por exemplo, dentre as causas foram listadas "Ação incorreta do Operador” e "Vazamento de etanol”, estes acontecimentos em conjunto com alguma faísca poderiam gerar a explosão e dentre as consequências estão "Danos a Pessoas" e "Dano ao meio ambiente" em decorrência do tamanho do desastre. 


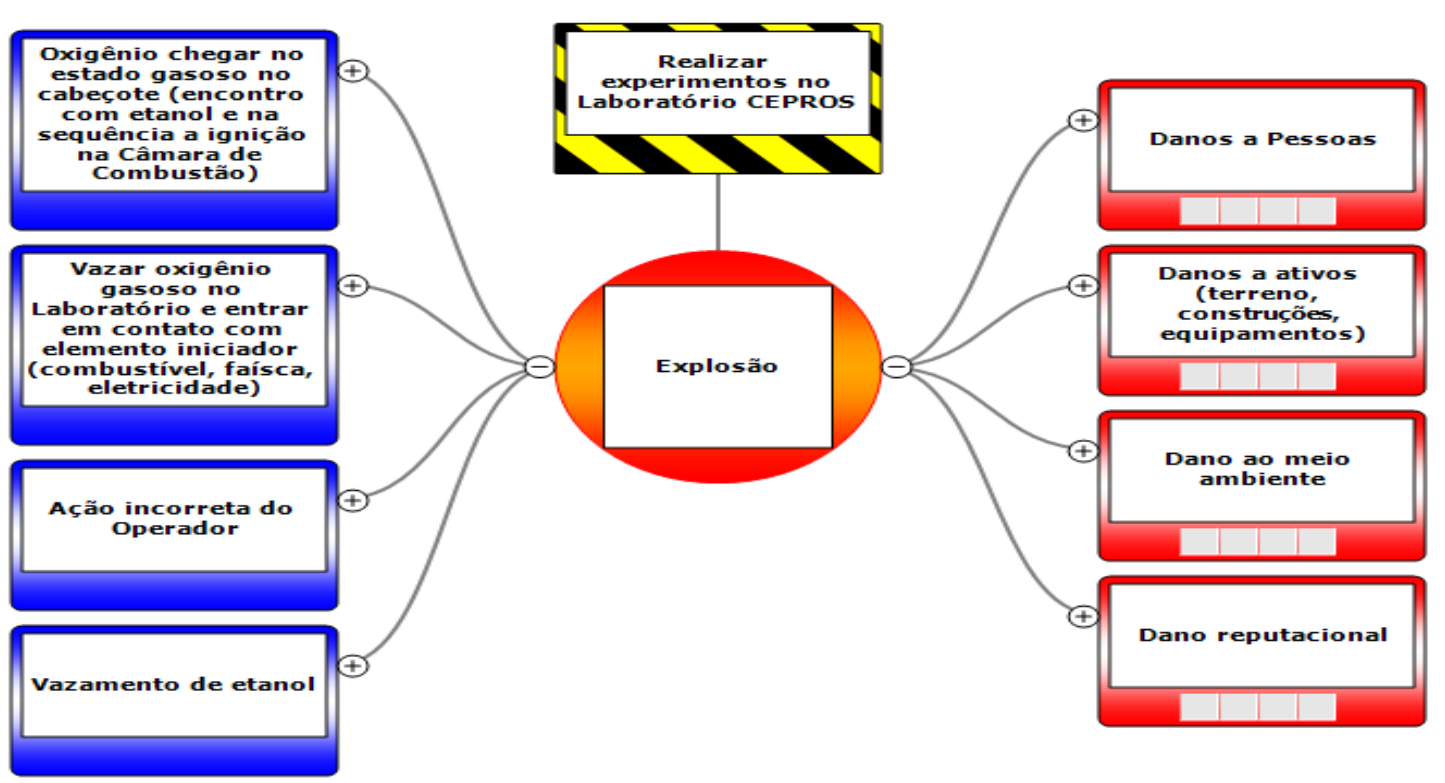

Figura 4 - Bowtie - Visão de Ameaças e Consequências

\section{CONCLUSÕES}

Com base no estudo realizado com a metodologia SSM, ao final foi constatado quais ações eram necessárias para a transformação da situação problemática (Alta exposição aos riscos no Laboratório. $\rightarrow$ Baixa exposição aos riscos no Laboratório; Baixa dedicação de professores e técnicos no planejamento de atividades do Laboratório. $\rightarrow$ Alta dedicação de professores e técnicos no planejamento de atividades do Laboratório).

Assim, pela SSM foram identificados os sistemas relevantes, as transformações e cada CATWOE, gerando ao final a listagem de ações de melhoria. Contudo, ainda se verificou a necessidade de análise dos perigos identificados principalmente na Figura Rica e respectivas barreiras ou ações de proteção, sendo aplicado nova avaliação a partir da utilização das metodologias de análise de perigos (HEMP e Bowtie). O resultado obtido, considerando os perigos potenciais às atividades do Laboratório, foi a identificação das ações para adoção de barreiras de controle e de mitigação como, por exemplo, plano de resposta as emergências, controle de acesso, treinamento de segurança de técnicos do laboratório, equipamentos adequados, sinalização de segurança e um programa de gerenciamento das operações do laboratório.

Este trabalho tratou da integração de métodos e aplicação para análise de um caso real de um problema complexo, contribuindo para segurança no Laboratório CEPROS. Como sugestões futuras, propõe-se a realização de uma interação da SSM, incluindo a perspectiva de mais atores, de modo a expandir a expressão do problema. Também se coloca a oportunidade de analisar outras transformações não incluídas neste estudo. Com relação a aplicação das transformações analisadas, é possível tanto estender a análise de perigos com as técnicas HEMP e Bowtie para mais eventos, bem como avaliar a efetividade da aplicação das soluções de barreiras propostas ao longo de sua implementação. 


\section{REFERÊNCIAS BIBLIOGRÁFICAS}

[1] F. T. Sakane, "Plano de Desenvolvimento Institucional 2011 - 2020," Ministério da Def. - Comando da Aeronáutica - Inst. Tecnológico Aeronáutica, vol. I, p. 175, 2011.

[2] S. R. Ensslin, "A incorporação da perspectiva sistêmico-sinergética na metodologia MCDA-Construtivista: uma ilustração de implementação," Florianópolis, SC, 2002.

[3] D. G. R. Sequeira, "Análise e avaliação de risco de incêndio através de diagramas 'Bow-Tie," Faculdade de Ciências e Tecnologia da Universidade Nova de Lisboa, 2010.

[4] S. A. Filho, V. P. Rabinovitz, F. de C. C. Fidalgo, L. G. T. de Menezes, and C. M. D. de Alcantara, "Análise de risco em Megaeventos esportivos, projeto UFBA na Copa," XXXIV Encontro Nac. Eng. Produção-ENEGEP, vol. 1, p. 15, 2014.

[5] ABNT NBR ISO 31000, Gestão de riscos - Diretrizes. ISO, 2018, p. 22.

[6] SSST, NR 9 - PROGRAMA DE PREVENÇÃO DE RISCOS AMBIENTAIS. 1994, p. 4.

[7] Conselho nacional de Educação - Câmara de Educação Superior, Resolução CNE/CES $n^{\circ}$ 11/2002, de 11 de Março de 2002. 2002, p. 4.

[8] B. A. de Felippes, J. G. Aguiar, and A. C. G. C. Diniz, "Sistema de Qualidade em laboratórios universitários: incentivo ao ensino, pesquisa e extensão," Revista de Ensino de Engenharia, vol. 30, no. 2, pp. 14-23, 03-Jun-2011.

[9] J. Simonsen, "Soft Systems Methodology - An Introduction." p. 18, 1994.

[10] S. Burge, "An Overview of the Soft Systems Methodology," System Thinking: Approaches and Methodologies. Rugby, Inglaterra, p. 14, 2015.

[11] I. Georgiou, "Managerial Effectiveness from a System Theoretical Point of View," Syst. Pract. Action Res., vol. 19, no. 5, pp. 441-459, Dec. 2006.

[12] I. Georgiou, "Making decisions in the absence of clear facts," Eur. J. Oper. Res., vol. 185, no. 1, pp. 299-321, Feb. 2008.

[13] SafetyHow, "What Is Hazard And Effect Management Process," SafetyHow, 2017. [Online]. Available:

http://www.safetyhow.com/index.php/What_Is_Hazard_And_Effect_Management_Pr ocess?action=print. [Accessed: 20-Nov-2017].

[14] G. Shaw, "The Hazard Risk Management Process," in Natural hazards analysis: reducing the impact of disasters, 1st ed., J. Pine, Ed. New York: CRC Press, 2009, pp. 193-220.

[15] A. de Ruijter and F. Guldenmund, "The bowtie method: A review," Saf. Sci., vol. 88, pp. 211-218, Oct. 2016.

[16] P. B. Checkland, Systems Thinking, Systems Practice, 1st ed. Chichester: John Wiley, Chichester, UK, 1981.

[17] R. Armson, Growing wings on the way: systems thinking for messy situations. Axminster: Triarchy Press, 2011.

[18] F. R. Parrilla, L. S. de A. Júnior, C. M. N. Belderrain, N. C. R. Bergiante, M. C. N. Belderrain, and N. C. R. Bergiante, "Estruturação do problema da baixa motivação do aluno em uma instituição de ensino superior privada," Rev. Gestão em Eng., vol. 5, no. 2 , p. 18, 2018. 